\title{
The design of Vehicle Low-speed intelligent braking system Fanbiao Bao ${ }^{1, a}$,Baoshan Huang ${ }^{2, b}$ \\ ${ }^{1,}$ aBeijing Institute of Technology, Zhuhai , Guangdong, 519085, China \\ abaofanbiao@126.com, bjetson_sbs@163.com
}

Keywords: Compound Processes, Machining, Ultrasonic, Electrochemical Finishing, Drilling, Surface Finish.

\begin{abstract}
This document explains and demonstrates how to prepare your camera-ready manuscript for Trans Tech Publications. The best is to read these instructions and follow the outline of this text. The text area for your manuscript must be $17 \mathrm{~cm}$ wide and $25 \mathrm{~cm}$ high (6.7 and 9.8 inches, resp.). Do not place any text outside this area. Use good quality, white paper of approximately $21 \mathrm{x} 29 \mathrm{~cm}$ or $8 \times 11$ inches (please do not change the document setting from A4 to letter). Your manuscript will be reduced by approximately $20 \%$ by the publisher. Please keep this in mind when designing your figures and tables etc.
\end{abstract}

\section{Introduction}

Generally speaking, traffic accidents caused by driver error is mainly due to the driver.Such as pilot error, drunk driving, braking is not timely and other human factors are the main reasons to cause the traffic accident. In the traffic congestion on the road, because of bad weather or the driver's carelessness and many other factors, even if the car at low speeds because of before and after the two car too close, and some drivers for your driving self feeling good, missed the best time for a vehicle brake, lead to the occurrence of rear end accident.

A lack of driving experience and inexperience is causing a major problem of traffic accident. Most of the traffic accident is a small automobile collision resulted in a busy road and small friction. The accident loss although rarely, but it is also a great loss of property to the driver. Car accidents are caused by a variety of factors such as mirror blind spots, drivers to judge the distance between obstacle incorrect.In view of the above circumstances, design an auxiliary driver active prevention of collision vehicle low speed intelligent braking system for driver assistant safe driving is necessary.

\section{The overall design scheme of the system}

This system uses AT89C51 MCU as the main controller. The system work process is as follows. The method adopted in the design of ultrasonic distance measurement to measure the distance of objects in front of the car. Firstly the ultrasonic transmitting probe emission signal and immediate feedback and reflection when the ultrasonic scanning to obstacles. Then the ultrasonic receiving probe receives the transmitted wave, ultrasonic receiving circuit to drive signals are transmitted to the single chip microcomputer.

It is only to display the distance when the obstacle is detected more than safe distance. It will start buzzer alarm if less than the safe distance. The implement agencies will began to work if reaching the braking distance and stepper motor drivies the brake pedal. The brake pedal movement simulation to generate force to achieve automatic brake aim. The design is shown in figure 1. 


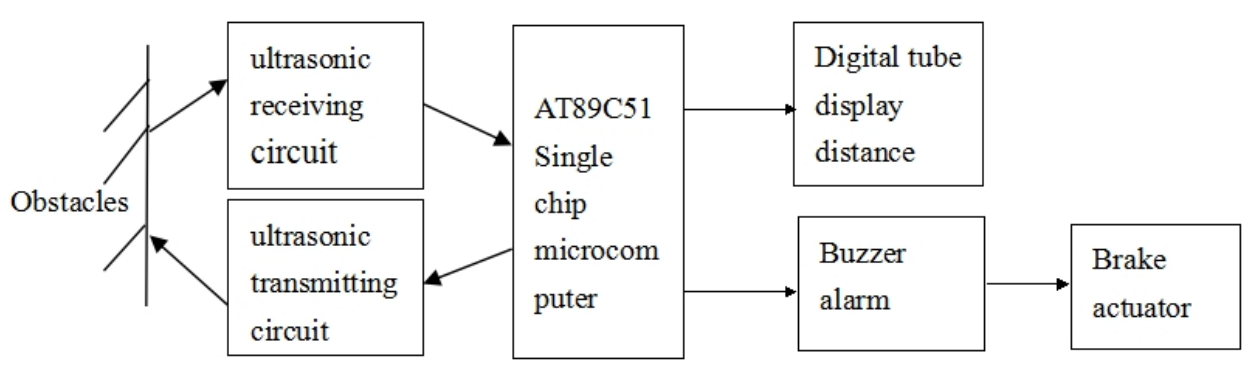

Fig.1 vehicle low speed intelligent braking system design

\section{Safety distance model}

Factors affecting the safe distance of vehicle size are as follows.

(1) effects of the vehicle operation speed, the higher the speed requires long safety distance;

(2) relative to the object near the front vehicle speed, safe distance is longer when thespeed is higher;

(3) the condition of the road, road friction coefficient smaller requires long safety distance;

(4) affect the car's weight;

(5) effects of vehicle braking performance, safe distance braking distance is greater demand for more. The vehicle braking performance is constant for the vehicle itself of the influence factors of safety distance. But the running speed and vehicle relative to the previous object near the velocity are changing.And the car encountered on the road condition is random variation.

\section{Vehicle braking distance}

The key lies in the intelligent brake is to establish reasonable and perfect alarm and safe distance braking model. In the design of this system adopted in a very short period of time, so do not consider the effect of acceleration, the vehicle before and after, as they are all uniform motions. This is a cycle detection mode. The time to the owners of the alarm signal and the automatic brake is directly affect the effects of collision. This whole system put forward a very high request system for data capacity and actuator operation single-chip processing speed. The following will be the system alarm analysis and determine the safe braking distance.

\section{Determine the braking distance}

The relative speed of the vehicles $\mathrm{V}$ and relative distance $\mathrm{S}$ are the real-time measurement of parameters of ultrasonic sensor. In the differential model, assuming the front vehicle motionless, the vehicle is moving at speed $\mathrm{V}$. At this time, the intelligent braking system to control the stepper motor to brake quickly, ignoring the brake operation time.So the braking distance can be calculate as folowing formula 1 .

$$
S_{0}=\frac{V^{2}}{2 a}
$$

( formula 1)

In formula 1 a is automotive brake maximum deceleration. Because of the difference in effects of auto speed on the tire performance of the resulting velocity on the brake and different materials of pavement also leads to different braking deceleration. The above factors set $\eta$ parameters for speed 
and $\mu$ for road adhesion coefficient. The revised model is as folowing formula 2 .

$$
S_{0}=\frac{V^{2}}{2 g\left(1-\eta V_{0}\right) \mu}
$$

( formula 2)

\section{Warning distance}

Warning distanceis the distance from the buzzer alarm to the driver to depresse the brake pedal brake application. The reaction time is the driver reaction time $\mathrm{T} 1$ and mechanical system $\mathrm{T} 2$. According to the braking distance data, the driver's reaction time T1 refers to the driver to identify obstacles, to put the pedal to the brake pedal force experienced by the time. This period is generally $0.3 \sim 1.0 \mathrm{~s}$. This design selects $\mathrm{T} 1=1.0 \mathrm{~s}$. The mechanical system reaction time is $\mathrm{T} 2$ to generate force applied to the brake pedal force, to produce the maximum braking deceleration time. This period is generally $0.2 \sim$ $0.9 \mathrm{~s}$. This design selects $\mathrm{T} 2=0.5 \mathrm{~s}$. So the alarm distance can be calculate as folowing formula 3 .

$$
S_{2}=S_{1}+V\left(T_{1}+T_{2}\right)
$$

( formula 3 )

According to the formula 2, formula 3 and formula 4 to calculate the theoretical data are shown in table 1.

Table 1. The theoretical data

\begin{tabular}{|c|c|c|c|c|c|c|c|c|}
\hline $\mathrm{V} / \mathrm{Km} \cdot \mathrm{h}-1$ & 5 & 10 & 15 & 20 & 30 & 40 & 50 & 80 \\
\hline $\begin{array}{c}\text { Braking distance S0 (m) } \\
\text { Safe braking distance } \\
\text { S1 (m) }\end{array}$ & 0.14 & 0.56 & 1.27 & 2.20 & 5.06 & 9.00 & $\begin{array}{c}12.3 \\
0\end{array}$ & 36.00 \\
\hline $\begin{array}{c}\text { Warning distance } \\
\text { S2 (m) }\end{array}$ & 2.24 & 4.79 & 7.64 & $\begin{array}{c}10.0 \\
0\end{array}$ & 18.07 & $\begin{array}{c}26.5 \\
6\end{array}$ & $\begin{array}{c}34.3 \\
6\end{array}$ & 72.92 \\
\hline
\end{tabular}

It can be see fron the Table 1 the vehicle speed is higher, the braking distance is longer.The car speed is high, the braking distance. When the vehicle speed reaches $20 \mathrm{Km} \cdot \mathrm{H}-1$, warning distance is up to $10 \mathrm{~m}$. The vehicle speed is higher, the emergency brake can produce undesirable effect is bigger to the vehicle and driver.So the system will avoid accidents by automatic braking operation when it detected a possible collision threat. When the speed below $15 \mathrm{~km}$ system can realize automatic brake. When the speed more than $15 \mathrm{~km}$ system can reduce the damage degree of collision.

\section{Conclusion}

This design is a new type of multifunctional automobile anti-collision warning system. It has the effect of early warning collision avoidance warning systems already on the market.The system has the function of new automatic brake. At present, the anti-collision used in the car on the car alarm system is mainly to send the reminding informationis. The domestic automobile factory mainly are concentrated in the surrounding environment detection, vehicle safety distance determination and alarm to warn the driver driving safety. This paper designed a car with automatic braking function of the automotive active collision avoidance system. The anti-collision system function is unchanged and increases the intelligent automobile brake functionc so that the automobile anti-collision alarm 
system to realize multiple functions.

Anti collision in the existing system is mainly based on the measurement results through the sound or image to inform the driver to change down around obstacles. Therefore, this paper adds auxiliary braking device automatically. The car can not rely on the driver automatically brake in order to achieve the purpose of safe driving. The design of the system use ultrasonic rangingon base of AT89C51 microcontroller as the core control unit.

\section{References}

[1] Guofu Liu,Qi Zhang,Jiulong Xiong,Xiufen Xie,Shan Peng.An Investigation of Calculation Method of Wheel Angular Acceleration in Anti-lock Braking System. International Conference on Information and Automation . 2008

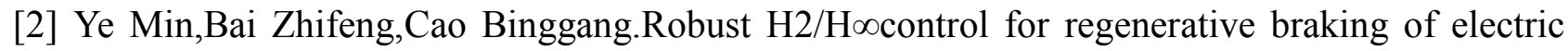
vehicles. 2007IEEE International Conference on Control and Automation . May30toJune12007

[3] Jie Shu,Weiwei Xiong.Effective regenerative braking control for smooth stop of a hybrid electric bus. IEEE (VPPC) . 2008

[4] Paul Caspi,Adrian Curic.From Simulink to SCAD/Lustre toTTA:a Llayered Approach for Distributed Embedded Applica-tions. . 2002

[5] Chen Fang PLC microcontroller remote control alarm system design [J]. occupation. 2010, (24). 